\title{
Optimal Cusum Control Chart for Censored Reliability Data with Log-logistic Distribution
}

\author{
B. Sadeghpour Gildeh, M. Taghizadeh Ashkavaey \\ Department of Statistics, Faculty of Mathematical Science \\ Ferdowsi University of Mashhad, Mashhad, Iran \\ E-mail: sadeghpour@um.ac.irm.taghizadeh@stu.umz.ac.ir
}

Received: 30 October 2014; revised: 09 October 2015; accepted: 20 October 2015; published online: 16 December 2015

\begin{abstract}
The goal of this work is to detect any potentially harmful change in a process. The reliability tests are assumed to generate type-I right-censored data following a log-logistic distribution with scale parameter $(\eta)$ and shape parameter $(\beta)$. For this purpose, we have constructed a likelihood ratio based simultaneous cumulative sum (CUSUM) control chart that targets changes in both the failure mechanism and the characteristic life (the simultaneous CUSUM chart for detecting shifts in the shape and the scale parameters). This control chart displays best performance for combinations with larger positive or negative shifts in the shape parameter, signaling on average in 5 samples in an out-of-control situation, while targeting an in-control average run length of 370. The simultaneous CUSUM chart's performance is highly dependent on the values of $\beta$, and on the interaction between them and the censoring rates and shift sizes.
\end{abstract}

Key words: Likelihood ratio, Type-I right-censoring, Log-logistic distribution, Cumulative sum (CUSUM) chart, Average run length

\section{INTRODUCTION}

Reliability data are typically censored (exact failure times are not known). The most common reason for censoring is the frequent need to analyze life test data before all units have failed. More generally, censoring arises when actual response values (e.g., failure times) cannot be observed for some or all units under study. Thus censored observations provide a bound or bounds on the actual failure times. Most reliability data are modeled using distributions for positive random variables like the exponential, weibull, gamma, lognormal and log-logistic. Relatively few applications use the normal distribution as a model for product life [1]. If one is interested in monitoring the quality and reliability characteristics of such processes, one needs to account for the challenges imposed by the nature of the data. We propose likelihood ratio based cumulative sum (CUSUM) control charts for censored lifetime data with non-normal distributions.

Hawkins and Olwell [2] observed that the optimal diagnostic for a step change in the parameter of any distribution is a cumulative sum (CUSUM) chart following with the score statistic

$$
z_{i}=\log \frac{L\left(y_{i} \mid \text { out }- \text { of }- \text { control }\right)}{L\left(y_{i} \mid \text { in }- \text { control }\right)},
$$

where $y_{i} ; i=1,2, \ldots$ is the $i$ th sample of size $n$ and $L$ is the likelihood function, formulated according to the distribution of choice and to the censoring scheme. Thus, $z_{i}$ is determined as the logarithm of the ratio between the likelihood of the data in the out-of-control and in-control scenarios, in the other word, $z_{i}$ is the likelihood ratio test statistic which is obtained by using Neyman-Pearson lemma. In this article, we consider optimal diagnostic for a step change in the parameter of any distribution is a cumulative sum (CUSUM) chart. In section II, we customize the score statistic $z_{i}$ for a likelihood ratio based CUSUM chart with samples of $n$ right-censored data. The CUSUM chart to monitor for shifts in both parameter of log-logistic lifetimes is provided in section III. In section $\mathrm{IV}$, we provide results of out-of-control performance of the simultaneous CUSUM chart. Finally, in section V, we present 
tables of simultaneous CUSUM chart for the shape and the scale parameters.

\section{CENSORING OF LIFETIME DATA}

Two aspects specific to lifetime data make the development of a control chart more demanding:

1. The data tend to be skewed and non-normally distributed.

2. The data typically are censored.

Right censoring is most common. It occurs whenever at least one item does not fail over the course of the test. Type-I right-censoring occurs when one stops the test at a certain predetermined time, and the testing of the remaining units that did not fail by that time is suspended.

In general, the likelihood function for any distribution including right-censored data is

$$
L(u, b, y)=\prod_{j=1}^{n} f\left(y_{j}\right)^{\delta_{j}}\left[1-F\left(y_{j}\right)\right]^{1-\delta_{j}},
$$

where

$$
\delta_{j}= \begin{cases}1 & \text { if item fails at time } \mathrm{t} \\ 0 & \text { if item is censored }\end{cases}
$$

and $\mathrm{f}$ and $\mathrm{F}$ are, respectively, the probability density function (pdf) and the cumulative distribution function (cdf) of the assumed distribution [2].

Using (1.1) and (2.1), the statistic $z_{i}$ for a single sample of $n$ observations becomes

$$
\begin{aligned}
z_{i} & =\log \frac{L\left(y_{i} \mid\left(\eta_{1}, \beta_{1}\right)\right)}{L\left(y_{i} \mid\left(\eta_{0}, \beta_{0}\right)\right)} \\
& =\log \frac{\prod_{j=1}^{n} f_{1}\left(y_{i j}\right)^{\delta_{i j}}\left[1-F_{1}\left(y_{i j}\right)\right]^{1-\delta_{i j}}}{\prod_{j=1}^{n} f_{0}\left(y_{i j}\right)^{\delta_{i j}}\left[1-F_{0}\left(y_{i j}\right)\right]^{1-\delta_{i j}}} \\
& =\log \prod_{j=1}^{n}\left[\frac{f_{1}\left(y_{i j}\right)}{f_{0}\left(y_{i j}\right)}\right]^{\delta_{i j}}\left[\frac{1-F_{1}\left(y_{i j}\right)}{1-F_{0}\left(y_{i j}\right)}\right]^{1-\delta_{i j}} \\
& =\sum_{j=1}^{n} \log \left[\frac{f_{1}\left(y_{i j}\right)}{f_{0}\left(y_{i j}\right)}\right]^{\delta_{i j}}+\sum_{j=1}^{n} \log \left[\frac{1-F_{1}\left(y_{i j}\right)}{1-F_{0}\left(y_{i j}\right)}\right]^{1-\delta_{i j}} \\
& =\sum_{j=1}^{n} \delta_{i j} \log \left[\frac{f_{1}\left(y_{i j}\right)}{f_{0}\left(y_{i j}\right)}\right] \\
& +\sum_{j=1}^{n}\left(1-\delta_{i j}\right) \log \left[\frac{1-F_{1}\left(y_{i j}\right)}{1-F_{0}\left(y_{i j}\right)}\right] .
\end{aligned}
$$

\section{1. Log-logistic distribution}

The log-logistic distribution is very useful in a wide variety of applications, especially in the analysis of survival data [3]. The log-logistic distribution is very similar in shape to the log-normal distribution; however, it has the advantage of having simple algebraic expressions for its survivor and hazard functions and a closed form for its distribution function. It is therefore more convenient than the log-normal distribution in handling censored data. However, due to the symmetry of the log-logistic distribution, it may be inappropriate for modeling censored survival date, especially for the cases where the hazard rate is skewed or heavily tailed.

The probability density function and cumulative distribution function of log-logistic distribution are given as follows [4]

$$
\begin{array}{r}
f(t)=\frac{\left(\frac{\beta}{\eta}\right)\left(\frac{t}{\eta}\right)^{\beta-1}}{\left[1+\left(\frac{t}{\eta}\right)^{\beta}\right]^{2}}, \\
F(t)=\left\{\begin{array}{lr}
{\left[1+\left(\frac{t}{\eta}\right)^{-\beta}\right]^{-1},} & t>0, \beta>1, \\
0, & \text { elsewhere, }
\end{array}\right.
\end{array}
$$

where $\eta>0$ and $\beta>0$ are parameters. Its survivor function and hazard function are, respectively [5]

$$
s(t)=1-F(t)=\left[1+\left(\frac{t}{\eta}\right)^{\beta}\right]^{-1},
$$

and

$$
h(t)=\frac{\left(\frac{\beta}{\eta}\right)\left(\frac{t}{\eta}\right)^{\beta-1}}{1+\left(\frac{t}{\eta}\right)^{\beta}}, \quad t>0 .
$$

\section{2. Detect simultaneous shifts in the shape parameter $\beta$, and in the scale parameter $\eta$ of the log-logistic} distribution

We adjust the likelihood ratio based CUSUM chart to monitor for shifts in the process, caused by both a shift in the shape parameter $\beta$, from $\beta_{0}$ to $\beta_{1}=\left(1-d_{\beta}\right) \times \beta_{0}$, and a shift in the scale parameter $\eta$, from $\eta_{0}$ to $\eta_{1}=\left(1-d_{\eta}\right) \times \eta_{0}$, where $\mathrm{d} \times 100 \%$ represents a percent change in $\eta$ and $\beta$. We generate samples of $n$ items in a testing process and $T$ represents the lifetime of the products. We stop the test at a predetermined time $C$. Any product that had not failed by time $\mathrm{C}$ generates a censored lifetime. By using Eq. (II.2), the statistic $z_{i}$ is given by

$$
\begin{aligned}
z_{i} & =\sum_{j=1}^{n} \delta_{i j} \log \frac{\beta_{1}}{\beta_{0}}+n \log \frac{\eta_{1}^{\beta_{1}}}{\eta_{0} \beta_{0}} \\
& +\left(\beta_{1}-\beta_{0}\right) \sum_{j=1}^{n} \delta_{i j} \log t \\
& +\sum_{j=1}^{n}\left(1-\delta_{i j}\right) \log \frac{\eta_{0}^{\beta_{0}}+t^{\beta_{0}}}{\eta_{0}^{\beta_{1}}+t^{\beta_{1}}} .
\end{aligned}
$$

\section{CUMULATIVE SUM CONTROL CHART}

In this section, we construct the CUSUM chart to monitor for shifts in both the scale parameter and the shape parameter of log-logistic lifetimes. The in-control values of the scale and shape parameters are assumed to be known. 


\section{1. Design the CUSUM chart}

The CUSUM chart, developed by E. Page [6] incorporates past information into each individually plotted observation. This increases the sensitivity of the CUSUM chart in detecting small shifts in the process. The cumulative sum (CUSUM) control chart has been widely used in modern industries to monitor process mean shifts. We consider two separate pieces of the Eq. (II.3):

$$
\begin{aligned}
k_{i}=- & \left(\sum_{j=1}^{n} \delta_{i j} \log \frac{\beta_{0}}{\beta_{1}}+n \log \frac{\eta_{0}^{\beta_{0}}}{\eta_{1} \beta_{1}}\right), \\
w_{i} & =\left(\beta_{1}-\beta_{0}\right) \sum_{j=1}^{n} \delta_{i j} \log t \\
& +\sum_{j=1}^{n}\left(1-\delta_{i j}\right) \log \frac{\eta_{0}^{\beta_{0}}+t^{\beta_{0}}}{\eta_{0}^{\beta_{1}}+t^{\beta_{1}}},
\end{aligned}
$$

where Eq. (III.1) is constancy value and Eq. (III.2) is variable in CUSUM chart statistics. If we are interested in detecting a decrease in both the scale parameter and the shape parameter of log-logistic lifetimes, therefore the chart statistics are given as follows [7]

$$
\begin{aligned}
c_{0}^{-} & =0, \\
c_{i}^{-} & =\min \left[0, c_{i-1}^{-}+w_{i}-k_{i}\right] . \quad i=1,2, \ldots
\end{aligned}
$$

The CUSUM signals if $c_{i}^{-}<-h^{-}$, when the chart indicates a decrease in both parameters. Similarly, if we want to detect a positive shift in both the scale parameter and the shape parameter of log-logistic lifetimes, we use the chart statistics

$$
\begin{aligned}
& c_{0}^{+}=0, \\
& c_{i}^{+}=\max \left[0, c_{i-1}^{+}+w_{i}-k_{i}\right] . \quad i=1,2, \ldots
\end{aligned}
$$

The chart signals if $c_{i}^{+}>h^{+}$, when the chart indicates an increase in both of the scale parameter and the shape parameter of log-logistic lifetimes, $h^{+}$is a decision parameter. Factor for determining decision Interval $\left[h^{-}, h^{+}\right]$which acts as a control limit. Its values depend on the value of the probability of type-I error or ARL0. Generally, $h^{+}$is defined as 5, although sometimes 4 is utilized and $h^{-}=-h^{+}$. The correlation between beta and eta is not necessarily positive.

\section{2. Criterion for performance evaluation}

The design of the conventional CUSUM chart involves evaluation of the control-chart performance based on average run length (ARL) $[8,9]$. The in-control ARL, which refers to the average number of observations from the incontrol process before a false out-of-control alarm is raised, is a measurement of the false-alarm rate. The out-of-control ARL, which is the average number of observations required to detect a specific mean shift, represents the detection power of the control chart. The best chart gives the lowest out-ofcontrol ARL (OARL).

\section{SIMULATION}

In this section we study the properties of the simultaneous chart that detects changes in both the scale and the shape parameters through simulations and we discuss the results.

\section{1. Simulation Description}

We evaluated the performance of the likelihood based CUSUM chart monitoring for simultaneous shifts in both parameters of a log-logistic distribution in the following scenarios [10]:

1. Sample size $n=3,5,10$ (The samples with 3, 5, and 10 size are repeated 10000 times to reach the favorite OARL and IARL).

2. Theoretical in-control censoring rates $\mathrm{pc}=5 \%, 50 \%$, $80 \%$,

$$
p_{c}=\left[1+\left(\frac{t}{\eta}\right)^{\beta}\right]^{-1} .
$$

3. In-control values for the shape parameter $\beta=\beta_{0}=$ $0.5,1,3,5$.

4. In-control value for the scale parameter $\eta=\eta_{0}=1$, without loss of generality, since the chart's design and performance is independent of the in-control values of the scale parameter.

5. Shift size for the scale parameter $d_{\eta}=$ $+5 \%,+20 \%,-5 \%,-20 \%$. The out-of-control $\eta$ value is then $\eta_{1}=\eta_{0} \times\left(1-d_{\eta}\right)$.

6. Shift size for the shape parameter $d_{\beta}=$ $+5 \%,+20 \%,-5 \%,-20 \%$. The out-of-control $\beta$ value is then $\beta_{1}=\beta_{0} \times\left(1-d_{\beta}\right)$.

7. Desired in-control average run length (IARL) is equal to 370 .

8. We run the simulations to obtain the threshold and then to evaluate the out-of-control performance for each scenario with 10,000 replications.

\section{2. Simulation Results}

We explore the behavior of the simultaneous chart for different censoring rates, in-control values of the shape parameter and sample size, and for small and large positive and negative shifts in both the shape parameter and the scale parameter. Next, Figures 1 through 8, as well as tables 1 through 3 , illustrate the performance of the simultaneous CUSUM chart for specific combinations of shifts in the scale and the shape parameters. Each panel of each figure displays the impact of different combinations of shifts in the scale parameter and in the shape parameter on the out-of-control performance of the charts designed for those combinations with low and 
high values of beta. Each panel corresponds to a certain censoring rate. All panels are based on a sample size of $n=5$. Tables 1 through 3 support the information from the figures with numerical examples. Figures 1 and 2 consider combinations of a positive shift in the scale parameter, accompanied by a positive shift in the shape parameter. Figures 3 and 4 consider a positive shift in the scale parameter and a negative shift in the shape parameter. Figures 5 and 6 summarize the results for combinations with negative shifts in the scale parameter and positive shifts in the shape parameter. Figures 7 and 8 consider combinations with a negative shift in the scale parameter and a negative shift in the shape parameter. Tables 1,2 , and 3 illustrate numerically the shift effects by $\beta_{0}$ value and for different censoring amounts.

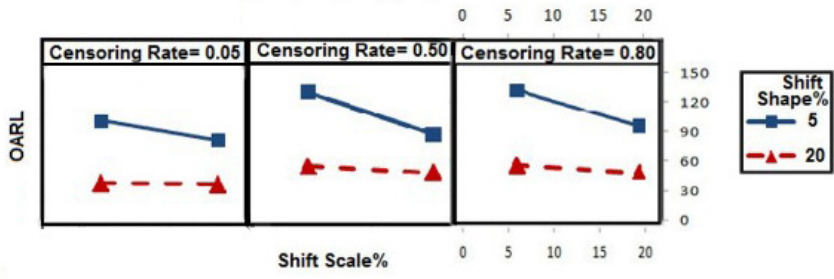

Fig. 1. Out-of-control performance of the simultaneous chart to detect an increase in the scale parameter and an increase in the shape parameter for $\beta_{0}=0.5$ and sample size $=5$

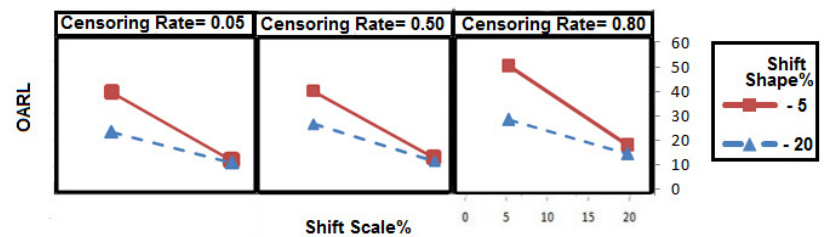

Fig. 2. Out-of-control performance of the simultaneous chart to detect an increase in the scale parameter and an increase in the shape parameter for $\beta_{0}=5$ and sample size $=5$

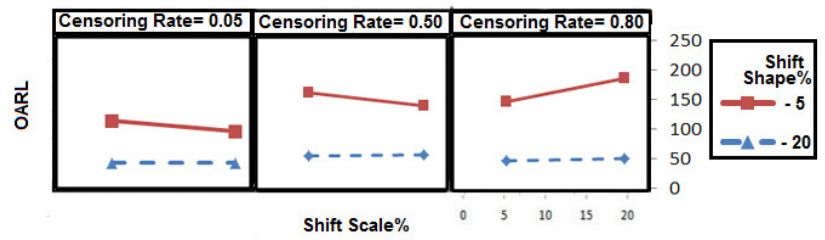

Fig. 3. Out-of-control performance of the simultaneous chart to detect an increase in the scale parameter and a decrease in the shape parameter for $\beta_{0}=0.5$ and sample size $=5$

In these figures and tables we examine the out-of-control performance in low, moderate, and high censoring scenarios and for different in-control failure mechanisms (modeled by $\beta_{0}$ ). We detail these behaviors for the intermediate sample size $n=5$. We notice that a $20 \%$ shift in the shape parameter, either positive or negative, in combination with any of the considered shifts in the scale parameter, result in the best chart performance, with any censoring rate. Here $\beta_{0}=0.5$. It appears that the magnitude of the shift in the shape parameter is an important performance driver. On the other hand, a $5 \%$ positive or negative shift in shape results in considerably poorer performance in the same scenario. A large in-control $\beta$ value $\beta_{0}=5$ mitigates the impact of the combination of shifts sizes and directions, especially for low and moderate censoring rates. As censoring rate increase the OARL increases accordingly. The chart's out-of-control performance is better for higher values of $\beta_{0}$. This conclusion can be drawn for values higher than 5 of $\beta_{0}$. There is a significant interaction effect between the in-control $\beta$ values, the censoring rates and the shift size and direction combinations. For example, from table 3 , when we expect about $80 \%$ data to be censored, the chart signals on average in 220 samples a simultaneous $20 \%$ negative shift in the scale parameter and $5 \%$ positive shift in the shape parameter when the in-control $\beta$ is $\beta_{0}=0.5$. On the other hand, the chart signals on average in 7 samples in the same scenario when $\beta_{0}=5$.

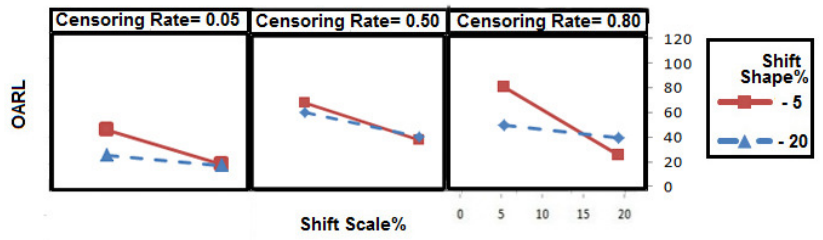

Fig. 4. Out-of-control performance of the simultaneous chart to detect an increase in the scale parameter and a decrease in the shape parameter for $\beta_{0}=5$ and sample size $=5$

Tab. 1. Out-of-control performance of the simultaneous CUSUM chart for $\beta_{0}=0.5$ and $\beta_{0}=5$, low censoring rate of $5 \%$, sample size $n=5$

\begin{tabular}{c|c|c|c}
\hline$\%$ Scale & \% Shape & OARL $\beta_{0}=0.5$ & OARL $\beta_{0}=5$ \\
\hline-20 & -20 & 18.8696 & 5.6975 \\
20 & -20 & 19.4082 & 7.1666 \\
-5 & -20 & 19.5911 & 16.3852 \\
5 & -20 & 19.4733 & 17.0915 \\
\hline-20 & 20 & 26.3436 & 5.3128 \\
20 & 20 & 27.0275 & 6.7992 \\
-5 & 20 & 28.2848 & 20.7731 \\
5 & 20 & 28.7084 & 21.5411 \\
\hline-20 & -5 & 74.6637 & 6.0021 \\
20 & -5 & 84.9149 & 7.8298 \\
-5 & -5 & 105.104 & 38.1665 \\
5 & -5 & 106.711 & 40.9919 \\
\hline-20 & 5 & 78.4067 & 5.8362 \\
20 & 5 & 87.364 & 7.5516 \\
-5 & 5 & 113.256 & 38.215 \\
5 & 5 & 114.363 & 40.7282 \\
\hline
\end{tabular}




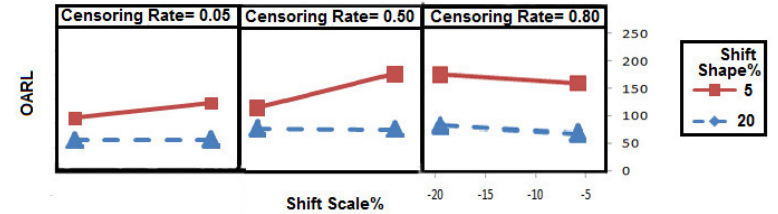

Fig. 5. Out-of-control performance of the simultaneous chart to detect a decrease in the scale parameter and an increase in the shape parameter for $\beta_{0}=0.5$ and sample size $=5$

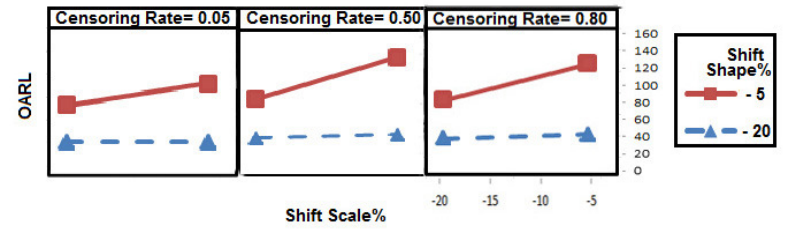

Fig. 7. Out-of-control performance of the simultaneous chart to detect a decrease in the scale parameter and a decrease in the shape parameter for $\beta_{0}=0.5$ and sample size $=5$

Tab. 2. Out-of-control performance of the simultaneous CUSUM chart for $\beta_{0}=0.5$ and $\beta_{0}=5$, moderate censoring rate of $50 \%$, sample size $n=5$

\begin{tabular}{c|c|c|c}
\hline$\%$ Scale & \% Shape & OARL $\beta_{0}=0.5$ & OARL $\beta_{0}=5$ \\
\hline-20 & -20 & 26.1644 & 5.7401 \\
20 & -20 & 37.7026 & 13.5246 \\
-5 & -20 & 31.3665 & 19.5428 \\
5 & -20 & 34.8824 & 43.6485 \\
\hline-20 & 20 & 59.3121 & 5.6492 \\
20 & 20 & 41.5908 & 7.3519 \\
-5 & 20 & 56.8183 & 34.898 \\
5 & 20 & 49.9723 & 25.2974 \\
\hline-20 & -5 & 78.9061 & 6.0791 \\
20 & -5 & 138.631 & 10.4381 \\
-5 & -5 & 135.306 & 38.8582 \\
5 & -5 & 167.683 & 55.6008 \\
\hline-20 & 5 & 107.019 & 6.0183 \\
20 & 5 & 92.6019 & 8.9417 \\
-5 & 5 & 184.566 & 46.2717 \\
5 & 5 & 146.802 & 41.5159 \\
\hline
\end{tabular}

Tab. 4. Simultaneous CUSUM chart for the shape parameter, $\% 20$ decrease in the scale parameter, in-control and out-of-control ARL for $\beta=\beta_{0}=0.5, \eta_{0}=1$

\begin{tabular}{c|c|c|c|c}
\hline Censoring rate & $\%$ Shape & Threshold $\mathrm{h}$ & IARL & OARL \\
\hline$\% 5$ & -5 & 2.43141 & 373.609 & 62.3474 \\
\hline$\% 50$ & -5 & 3.2485 & 374.424 & 66.5942 \\
\hline$\% 80$ & -5 & 3.19512 & 371.959 & 68.0452 \\
\hline$\% 5$ & -20 & 3.41332 & 372.307 & 16.6114 \\
\hline$\% 50$ & -20 & 5.31464 & 372.881 & 26.2892 \\
\hline$\% 80$ & -20 & 5.92879 & 371.788 & 24.8298 \\
\hline
\end{tabular}

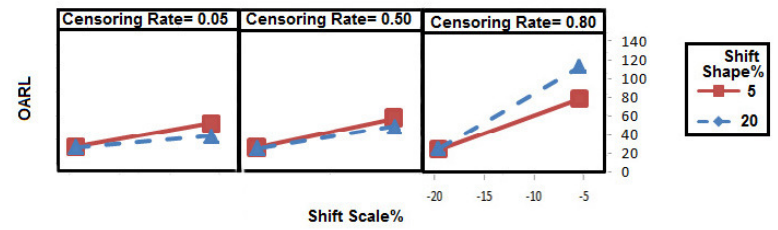

Fig. 6. Out-of-control performance of the simultaneous chart to detect a decrease in the scale parameter and an increase in the shape parameter for $\beta_{0}=5$ and sample size $=5$

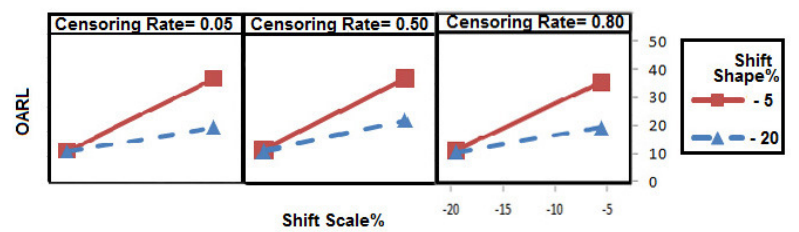

Fig. 8. Out-of-control performance of the simultaneous chart to detect a decrease in the scale parameter and a decrease in the shape parameter for $\beta_{0}=5$ and sample size $=5$

Tab. 3. Out-of-control performance of the simultaneous CUSUM chart for $\beta_{0}=0.5$ and $\beta_{0}=5$, high censoring rate of $80 \%$, sample size $n=5$

\begin{tabular}{c|c|c|c}
\hline$\%$ Scale & \% Shape & OARL $\beta_{0}=0.5$ & OARL $\beta_{0}=5$ \\
\hline-20 & -20 & 25.846 & 5.9859 \\
20 & -20 & 40.9191 & 41.6452 \\
-5 & -20 & 31.6003 & 19.4964 \\
5 & -20 & 35.011 & 57.2864 \\
\hline-20 & 20 & 76.2435 & 8.1025 \\
20 & 20 & 39.2892 & 11.4766 \\
-5 & 20 & 55.7596 & 119.37 \\
5 & 20 & 48.3591 & 27.4689 \\
\hline-20 & -5 & 81.0333 & 6.9846 \\
20 & -5 & 236.178 & 20.1833 \\
-5 & -5 & 132.115 & 43.4878 \\
5 & -5 & 177.893 & 106.096 \\
\hline-20 & 5 & 220.72 & 7.5589 \\
20 & 5 & 98.7429 & 15.4725 \\
-5 & 5 & 196.936 & 78.9923 \\
5 & 5 & 142.699 & 53.2435 \\
\hline
\end{tabular}

\section{SIMULATION RESULTS FOR THE SIMULTANEOUS CHART FOR THE SHAPE AND THE SCALE PARAMETERS}

In this section we describe the results given in tables 4 and 5, for each considered in-control value of the shape parameter, censoring rate, sample size and the smallest shifts size to be detected, the parameters of the CUSUM chart design: the threshold $h$ found through trial-and-error, the estimated average in-control run length and the estimated average outof-control run length are calculated. If there is a 5\% negative 
shift in the shape parameter and a $20 \%$ negative shift in the scale parameter, with censoring rates of $5 \%, 50 \%$ and $80 \%$, in a scenario with sample size $n=10, \beta_{0}=0.5$ and $\beta_{0}=5$, respectively the simultaneous chart detects such a combined shift on average in 62, 66, 68 and 4, 5, 9 samples, whereas $20 \%$ negative shift in the shape parameter and a $20 \%$ negative shift in the scale parameter, with same conditions, respectively the simultaneous chart detects such a combined shift on average in 16, 26, 24 and 4, 4, 7 samples, when the chart is designed for this scenario to achieve an in-control average run length of 370 .

Tab. 5. Simultaneous CUSUM chart for the shape parameter, $\% 20$ decrease in the scale parameter, in-control and out-of-control ARL for $\beta=\beta_{0}=5, \eta_{0}=1$

\begin{tabular}{c|c|c|c|c}
\hline Censoring rate & $\%$ Shape & Threshold h & IARL & OARL \\
\hline$\% 5$ & -5 & 4.49295 & 373.853 & 4.8323 \\
\hline$\% 50$ & -5 & 4.83178 & 370.225 & 5.5847 \\
\hline$\% 80$ & -5 & 4.78027 & 373.36 & 9.3696 \\
\hline$\% 5$ & -20 & 4.61965 & 370.422 & 4.3435 \\
\hline$\% 50$ & -20 & 5.87886 & 373.526 & 4.6483 \\
\hline$\% 80$ & -20 & 5.94266 & 395.879 & 7.101 \\
\hline
\end{tabular}

\section{CONCLUSION}

The goal of this work was to address a number of challenges in the area of monitoring reliability characteristics of lifetime data. Lifetime data resulting from lifetime tests usually are censored, involve multiple items on test, and follow non-normal distributions. The log-logistic distribution enjoys popularity with in reliability practice, given its flexibility to model a variety of failure mechanisms. We focused on the paradigm of samples of $n \geq 1$ products on a test stand. High values of $\beta_{0}$ result in good performance of the simultaneous chart and mitigate the effect of the other performance drivers: the censoring rate, the sample size, and the types of shifts. This research introduces a more comprehensive monitoring procedure for the log-logistic process with right-censored data, meant to bring insight into what type of changes can occur and what is the out-of-control performance of the monitoring procedures in each circumstance. We have also provided detailed guidelines for the practitioner to develop customized charts.

\section{References}

[1] W. Q. Meeker and L. Escobar, Statistical Methods for Reliability Data, John Wiley and Sons, New York, NY 1998.

[2] D. M. Hawkins and D. H. Olwell, Cumulative Sum Charts and Charting for Quality Improvement, Springer-Verlag, New York, NY 1998.

[3] J. O'Quigley and L. Struthers, Survival models based upon the logistic and log-logistic distributions, Computer Programs in Biomedical Researches 15, 3-12 (1982).

[4] A. Ragab and J. Green, On order statistics from the log-logistic distribution and their properties, Commun. Statist, Theory and Meth. 13. 2713- 2724 (1984).

[5] S. Bennett, Progressive Censoring: Theory, Methods and Loglogistic regression model for survival data, Applied Statistics 32, 165-171 (1983).

[6] E. Page, Continuous Inspection Schemes, Biometrika 41. 100115 (1954).

[7] D.C. Montgomery, Introduction to Statistical Quality Control, 5th edition, John Wiley and Sons, New York, NY 2004.

[8] G. Moustakides, Optimal Stopping Times for Detecting Changes in Distributions, The Annals of Statistics 14(4), 13791387 (1986).

[9] A. F. Bissell, CUSUM techniques for quality control (with discussion), Applied Statistics 18, 1-30 (1969).

[10] D. A. Olteanu, Cumulative Sum Control Charts for Censored Reliability Data, PhD thesis Faculty of the Virginia Polytechnic Institute and State University, Blacksburg, Virginia (2010).

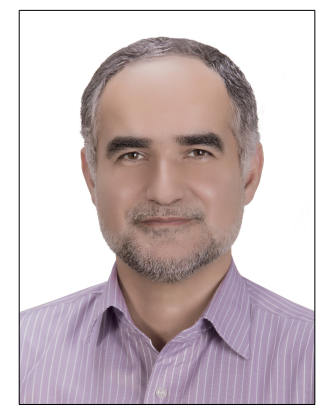

Bahram Sadeghpour Gildeh is an associate professor of Statistics at the Ferdowsi University of Mashhad, Iran. He received his MSc in Mathematical Statistics at the Ferdowsi University of Mashhad (IRAN) and PhD in informatics at the Blaise Pascal University (FRANCE). His research interests include Statistical Quality Control, Capability Analysis, and Fuzzy Statistics. 


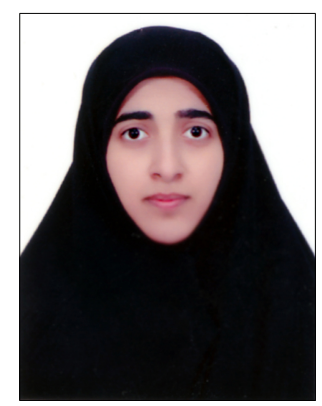

Maryam Taghizadeh received her BSc in Statistics at the University of Guilan and her MSc at the University of Mazandaran in Statistics (IRAN). Her MSc dissertation title: Cumulative Sum Control Charts for Censored Data. Her research interests include Statistical Quality Control, Censored data analysis and Statistical methods 